\title{
Prognostic IMPORTANCE OF CYCLIN E 1 EXPRESSION IN NEUROBLASTIC TUMORS IN CHILDREN
}

\author{
Katarzyna Taran ${ }^{1}$, Agata Owecka², Józef Kobos ${ }^{2}$
}

1Department of Pathology, Medical University of Lodz, Lodz, Poland

2Department of Pathology of the Age of Development, Konopnicka Memorial Hospital, Medical University of Lodz, Lodz, Poland

\begin{abstract}
A number of studies have indicated that cyclin E plays an important role in a variety of neoplastic processes.

In our study we evaluated cyclin E1 expression and the possible prognostic value of this protein in neuroblastic tumors in children..

Cyclin E1 expression was investigated by means of immunohistochemical analysis of 25 neuroblastic tumor tissue samples.

We found a significant correlation between high cyclin E1 expression and deaths due to neoplastic disease. The mean values of cyclin $\mathrm{E} 1$ indexes in fatal cases were twice as high as in other cases.

The results indicate that high cyclin E1 expression may have prognostic importance in neuroblastic tumors in children.
\end{abstract}

Key words: neuroblastoma, childhood, cyclin E, prognosis.

\section{Introduction}

Neuroblastoma is the most common extracranial neoplasm of the age of development, but its biological behavior still remains unknown. It is a malignant tumor with prognosis varying from very favorable to unfavorable depending on the patient's age, the clinical stage of disease as well as morphological structure of the tumor [1-4]. It is estimated that $8-10 \%$ of all malignant neoplasms in patients under 15 years of age are neuroblastic tumors and they are responsible for $15 \%$ of fatalities among children treated for malignancies [5-8].

The first findings indicative of the genetic etiology of neuroblastic tumors date back to 1983 when the concomitance of MYCN oncogene amplification and overexpression of $B$ receptor of tyrosine kinase (TrkB) were detected in patients with tumors of the neuroblastoma type. Some authors pose the hypothesis that neuroblastoma incidence is higher among individuals with a risk factor of neurofibromatosis type $1(\mathrm{NF} 1)$ as well as with Hirschsprung's disease [9].
Neuroblastic tumors originate from the neural crest of the sympathetic nervous system, and comprise neuroblasts of divergent degrees of maturity, stem and stromal cells. Most primary neuroblastic tumors (65\%) are situated in the abdominal cavity. Half of them develop in the adrenal medulla. Neuroblastoma may also appear in the cervical, thoracic and pelvic areas [10]. Microscopically neuroblastoma belongs to the group of small round cell tumors. The ones with a favorable prognosis are made up of clusters of mature neurons surrounded by a dense stroma of Schwann cells. Ganglioneuroblastomas contain both mature cells that manifest ganglion cell morphology and undifferentiated neuroblasts. As far as the tumors with the highest degree of risk are concerned, small blue cells with scarce cytoplasm and hyperchromatic nuclei take precedence in the microscopic picture $[4,11]$.

First and foremost, prognosis relies on the clinical stage of the disease, the age of the child, and the presence of $\mathrm{N}$-myc oncogene copies as well as of other markers such as TrkA receptor for either nerve growth factor (NGF) or ferritin level [2]. New prognostic markers are still be- 
ing sought. It has recently been noted that cyclin E1 may play a significant role in neoplastic processes. The molecules of cyclin E participate in the regulation of the cell cycle by controlling the kinases dependent upon them, predominantly CDK2 as well as, to a lesser degree, CDK1 and CDK3. The "incorporation" of cyclins into the chromatin chain occurs during the G1 phase of the cell cycle and lasts until the end of phase $\mathrm{S}$. Cyclin $\mathrm{E}$ is also involved in the process of DNA replication initiation as well as controlling genomic stability [12-15].

Cyclin E1 belongs to the IgG2a class, $\kappa$. Overexpression of cyclin $\mathrm{E} 1$ reduces the duration of the cell cycle G1 phase, accelerating the onset of phase S. Like in all other cyclins, its activation is mainly dependent on kinase CDK while its activity is regulated by suppressor proteins such as p16. Between the phases G1 and $\mathrm{S}$ of the cell cycle there is the so-called restriction point " $R$ " that is a critical point suitable for the growth control of the cells. Disturbances that affect it may lead to the uncontrolled overexpression of particular factors, including cyclin $\mathrm{E}$, with the resulting neoplasm development. The locus of cyclin $\mathrm{E}$ is situated at (19q12q13) and tends to appear in multiple copies in cases of human tumors [15-17].

The aim of the study was to carry out a morphological assessment of the expression of cyclin E1 and evaluate its possible prognostic value in neuroblastic tumors in children.

\section{Material and methods}

Twenty-five tissue samples of neuroblastic tumors from the archives of the Department of Pathology of the Age of Development, Medical University of Lodz, were included in the study. The tissue materials were fixed in $10 \%$ buffered formalin for 24-48 hours and embedded in paraffin blocks. The blocks were then cut into 3-4 micrometer sections and stained with hematoxylin and eosin (HE) and also used in immunohistochemical research with the use of mouse monoclonal antibody against cyclin $\mathrm{E}$ clone $13 \mathrm{~A} 3$ in dilution $1: 50$ (Novocastra Laboratories Ltd.). The staining was performed following an endogenous peroxidase blocking procedure $(0.5 \%$ hydrogen peroxide/methanol for 10 minutes) and high temperature antigen unmasking technique $(0.01 \mathrm{M}$ citrate buffer, $\mathrm{pH}$ 6.0). The sections were incubated with anticyclin $\mathrm{E}$ antibody for 1 hour at room temperature followed by mouse Envision + HRP detection system (DAKO) for $30 \mathrm{~min}$. Peroxidase reaction was developed using liquid $\mathrm{DAB}$ (DAKO). The negative controls were sections in which initial antibodies were replaced with TBS buffer and the further procedure was identical with the standard immunohistochemical procedure. The positive controls were sections that showed a strong positive reaction towards the antigens examined, verified in advance. The percentage of nuclei stained in tumor components was assessed by a computer image analysis system (Mul- ti Scan Base v. 8.08 - Computer Scanning System, Ltd). The microscopic picture was transferred to the computer memory by means of a video camera (CC2OP) coupled with a microscope (Nikon Microphot FXA).

A nuclear type of reaction with brown coloration of neoplastic cell nuclei was interpreted as a positive reaction. The reaction was evaluated in the sites of the preparation (visible areas) that showed the largest concentrations of the marker, the so-called hot spots of the tumor. Cyclin E1 expression (the percentage of cyclin E1-positive tumor cells) was derived by counting at least 1000 tumor cells. In statistical analysis there were also investigated the correlations between the expression of cyclin E1 and widely accepted prognostic factors in the neuroblastoma group of tumors (histological type, stage, age) as well as selected histoclinical features (sex, presence of metastases, recurrence and number of deaths due to neoplastic disease). For statistical analysis of results we used the statistical package SYSTAT for Windows (Version 5.03, SYSTAT, Inc). For all measurable features, the mean and median values were established. For the estimation of differences in examined groups we used the Kruskal-Wallis or MannWhitney test. In subgroups with less than 5 cases we used Fisher's test and/or Yates' correction. The difference was considered statistically significant if $\mathrm{p}<0.05$.

\section{Results}

\section{Histoclinical features}

The study group of neuroblastic tumor tissue samples comprised 25 cases. Twelve tumors were obtained from girls (48\%), 13 from boys (52\%). The youngest patient was 1 month old while the oldest was 151.5 months old. The mean age of children upon diagnosis was 37.9 months, with the median at 24.7 and standard deviation at 36.1. Among 25 evaluated patients, 7 died of the disease (28\%), 12 were found to suffer from metastasis (48\%), and 6 (24\%) from recurrences. All patients were subjected to surgical treatment and chemotherapy with 10 administered radiotherapy (40\%). The most numerous group comprised patients diagnosed in the $4^{\text {th }}$ stage of the disease (10 patients; $40 \%$ ), followed by those in the $3^{\text {rd }}$ stage ( 6 patients; $24 \%$ ), in the $2^{\text {nd }}$ stage ( 5 patients; $20 \%$ ), and in the $1^{\text {st }}$ stage ( 4 patients; $16 \%$ ).

In microscopic examination of investigated tumor tissue samples, the following histological types of tumor were identified: poorly differentiated neuroblastoma (9 cases; $36 \%$ ), differentiating type neuroblastoma (10 cases; $40 \%$ ), ganglioneuroblastoma ( 3 cases; $12 \%)$ and ganglioneuroma ( 3 cases; $12 \%$ ).

\section{Immunohistochemical studies and statistical analysis}

We found cyclin E1 expression in all the examined tumor tissue samples. The mean value of cyclin $\mathrm{E} 1$ in- 
dex was 22.537 (with the minimum at 10.200, the maximum at 60.000, the standard deviation at 12.799, and the median at 16.800). The microscopic picture of cyclin $\mathrm{E} 1$ expression in neuroblastoma is shown in Fig. 1 and Fig. 2. A higher value of cyclin E1 index proved characteristic of neoplasms with unfavorable histology. The lowest indexes were observed in ganglioneuromas, the highest in poorly differentiated neuroblastomas. The cyclin E1 indices in the poorly differentiated and differentiating neuroblastomas taken together as a group were found to be nearly twice as high $(23.4 \%)$ as in the ganglioneuroma group alone (15.3\%). However, no statistically significant relations were revealed upon the analysis of the results. The mean values of cyclin E1 index in deceased children was twice as high $(36.0 \%)$ as in the remainder of cases $(17.7 \%)$. Five of nineteen children with tumors with high cyclin E1 expression died due to neoplastic disease. We observed a statistically significant correlation between high indexes of cyclin E1 expression and death of the patients due to neoplastic disease $(\mathrm{p}=0.007)$. The values of cyclin E1 index were higher in cases with metastases $(29 \%)$ than in the remainder of cases $(17 \%)$. Similar results were obtained upon the analysis of the values of cyclin E1 index with respect to recurrence incidence. In children suffering from recurrence the mean value of cyclin E1 index was higher $(28 \%)$ than in other cases $(21.3 \%)$, but the differences were not statistically significant.

Higher indices of cyclin E1 were observed at more advanced stages of the disease as follows: $4^{\text {th }}-32.6 \%$; $3^{\text {rd }}-17.6 \% ; 2^{\text {nd }}-13.0 \% ; 1^{\text {st }}-10 \%$, but without statistical significance.

\section{Discussion}

Solid tumors in children constitute one of the major problems of current pediatric oncology. Following injuries and accidents, neoplastic diseases are the main cause of mortality among children over two years of age. In modern medicine a cure may be found in as

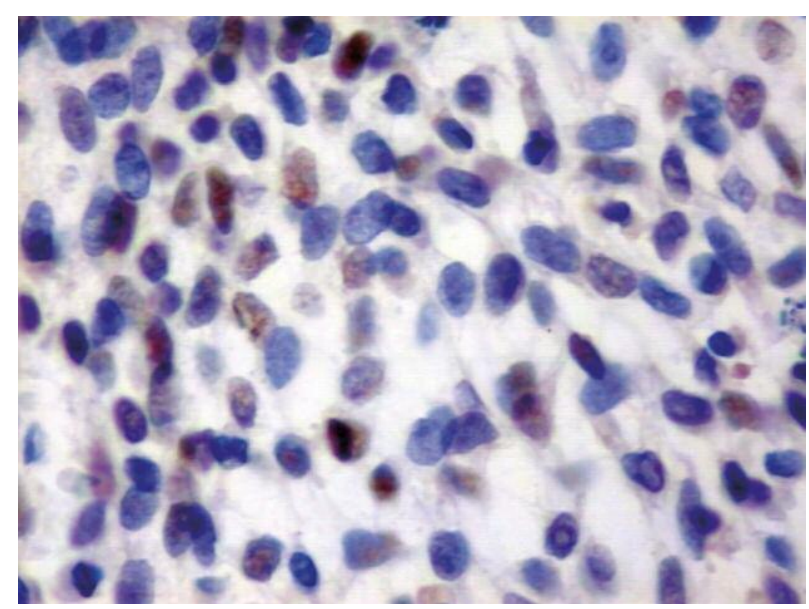

Fig. 1. Cyclin E1 expression in poorly differentiated neuroblastoma. Original magnification $400 \times$ many as $70 \%$ of pediatric cases by virtue of both stateof-the-art diagnostic techniques spanning laboratory, imaging and histological techniques that lead to reducing the time needed to establish the final diagnosis and novel therapeutic guidelines. The assessment of the presence of cell cycle-related proteins may yield important information about the biological behavior of a tumor. Because not all of the patients with neuroblastoma responded well to chemotherapeutic treatment some other than already known factors must take part in the course of neoplastic disease. The present study was carried out to investigate whether cell activity represented by cyclin E1 expression exists and to evaluate its possible prognostic value in neuroblastic tumors. Cyclin E constitutes protein molecules that serve as regulators within eukaryotic cells. Their activation involves kinase CDK2. According to the current literature, the overexpression of cyclin $\mathrm{E}$ in the cell nucleus is known to lead to shortening of the G1 phase of the cell cycle.

Cyclin E is considered to play a role in neoplastic processes as it disrupts the duration of particular cell cycle phases, giving way to genetic instability $[12-14,18]$.

The present study demonstrated that high indices of cyclin E1 are observed in the cells of neoplasms demonstrating low differentiation and thus of higher malignancy.

We observed higher values of cyclin E1 indices in potentially low differentiated types such as poorly differentiated, differentiating type and ganglioneuroblastoma rather than in ganglioneuroma. The values of cyclin E1 indices were higher also in cases showing a more clinically advanced stage of the disease. It was proved that the values of cyclin indices were considerably higher in the case of neuroblastomas than ganglioneuromas. In all likelihood, high values of cyclins $\mathrm{D}$ and $\mathrm{E}$ indices are associated with poor prognosis for patients with ganglioneuromas [19].

The studies evaluating Wilms' tumor tissue samples (in stromal, blastemal and epithelial components) re-

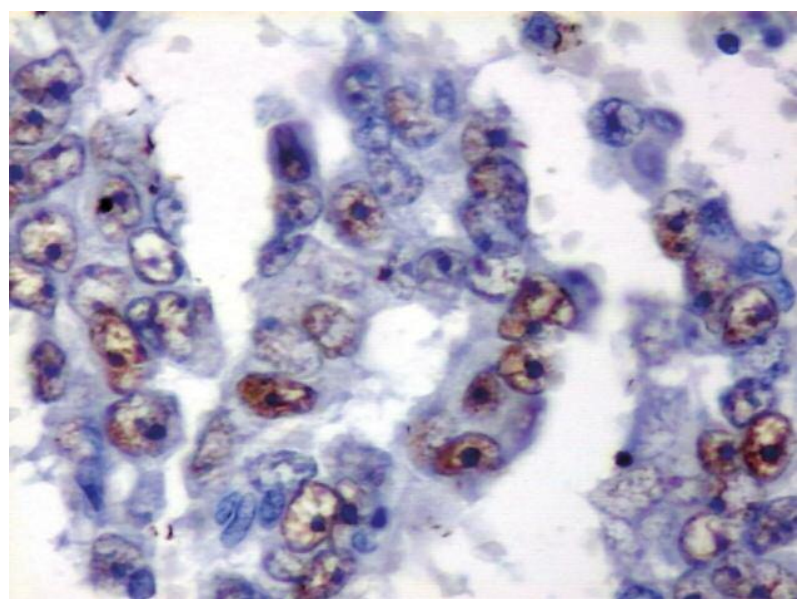

Fig. 2. Cyclin E1 expression in differentiating neuroblastoma. Original magnification $600 \times$ 
vealed correlations between high values of cyclin $\mathrm{E}$ and $\mathrm{Ki}-67$ indices, the presence of metastases and more "aggressive" (more resistant to chemotherapy) forms of nephroblastoma. The results proved that high values of cyclin $\mathrm{E}$ index occur in tumors with a higher histological risk and accompanying poor prognosis [20]. In animal models it was shown that high values of cyclin $\mathrm{E}$ index in mice caused genetic instability due to chromosome intervals and translocations. Furthermore, the researchers found that overexpression of cyclin $\mathrm{E}$ was coupled with p53 gene deficiency which further amplified murine neoplastic progression. The in vitro study by the same researchers indicated that overexpression of cyclin $\mathrm{E}$ raised the likelihood of murine Ras-dependent pulmonary cancer to a greater degree than the deficit of gene p53 [18].

There was also an article reporting that overexpression of cyclin $\mathrm{E}$ is due to neoplastic processes [13]. The authors mentioned that kinase-independent function of cyclin E was conducive to chromosomal instability and induced cancer cell proliferation. It was also underlined in the literature that the CDK-independent function of cyclin E may be a trigger for the "approval" for the replication and oncogenic transformation of cells [14]. An aspect of cyclin E still remains not completely known - the kinase-independent effect of cyclin $\mathrm{E}$ that leads to the disturbance of phase $\mathrm{G1}$, and is responsible for rendering cyclin $\mathrm{E}$ a marker associated with oncogenesis. Such information is especially important for studies on some malignancies in which despite many efforts we did not achieve expected results of performed treatment as with neuroblastoma. Results of numerous new studies created an unexpected picture of neuroblastic tumor biology and showed the possible prognostic impact of new markers [21, 22].

In summary, we have reported that there was an increase in cyclin E1 expression in low differentiated neuroblastic tumors which correlated with death due to neoplastic disease. These results support the conclusion that cyclin E1 expression may be used for assessing the proliferative activity and tumor cell dynamics and may indicate a prognostically unfavorable role of cyclin $\mathrm{E}$ in neuroblastic tumors.

Supported by: Medical University of Lodz Grant (50218-526) and European Union (EFS, ZPORR Program 2.6). The authors declare no conflict of interest.

\section{References}

1. Schor N. Neuroblastoma. Drug Discovery Today: Disease Models 2006; 3: 387-390.

2. Stefanowicz J, Iżycka-Świeszewska E, Drożyńska E, et al. Neuroblastoma and opsoclonus-myoclonus-ataxia syndrome clinical and pathological characteristics. Folia Neuropathol 2008; 46: 176-185.

3. Iżycka-Świeszewska E, Brzeskwiniewicz M, Woźniak A, et al EGFR, PIK3CA and PTEN gene status and their protein prod- uct expression in neuroblastic tumours. Folia Neuropathol 2010; 48: $238-245$

4. Hildebrandt T, Traunecker H. Neuroblastoma: A tumor with many faces. Current Paediatrics 2005; 15: 412-420.

5. Tononi G.P, Romani M. Genetic and epigenetic alterations in neuroblastoma. Cancer Lett 2003; 197: 69-73.

6. Noesel MM, Versteeg R: Pediatric neuroblastomas: genetic and epigenetic „Dance Macabre“. Gene 2004; 325: 1-15.

7. Goldsmith KC, Hogarty MD. Targeting programmed cell death pathways with experimental therapeutics: opportunities in high-risk neuroblastoma. Cancer Lett 2005; 228: 133-141.

8. Maris JM, Hogarty MD, Bagatell R, Cohn SL. Neuroblastoma. Lancet 2007; 369: 2106-2120.

9. Tonini GP, Longo L, Coco S, Perri P. Familial neuroblastoma: a complex heritable disease. Cancer Lett 2003; 197: 41-45.

10. Ross RA, Biedler JL, Spengler BA: A role for distinct cell types in determining malignancy in human neuroblastoma lines and tumors. Cancer Lett 2003; 197: 35-39.

11. Chlenski A, Liu S, Cohn SL. The regulation of angiogenesis in neuroblastoma. Cancer Lett 2003; 197: 47-52.

12. Möröy T, Geisen C. Cyclin E. Int J Biochem Cell Biol 2004; 36: 1424-1439.

13. Geng Y, Lee YM, Welcker M, et al. Kinase-independent function of cyclin E. Mol Cell 2007; 25: 127-139.

14. Zhang H: Life without Kinase: Cyclin E Promotes DNA Replication Licensing and Beyond. Molecular Cell 2007; 25: 175-176.

15. Payton M, Coats S: Cyclin E2, the cycle continues. Int J Biochem Cell Biol 2002; 34: 315-320.

16. Roberts JM, Sherr CJ. Bared essentials of CDK2 and cyclin E. Nat Genet 2003; 35: 9-10.

17. Coqueret O. Linking cyclins to transcriptional control. Gene 2002; 299: 35-55.

18. Loeb KR, Kostner H, Firpo E, et al. A mouse model for cyclin E-dependent genetic instability and tumorigenesis. Cancer Cell 2005; 8: 35-47

19. Molenaar JJ, Ebus ME, Koster J, et al. Cyclin D1 and CDK4 activity contribute to the undifferentiated phenotype in neuroblastoma. Cancer Res 2008; 68: 2599-2609.

20. Berrebi D, Leclerc J, Schleiermacher G, et al. High cyclin E staining index in blastemal, stromal or epithelial cells is correlated with tumor aggressiveness in patients with nephroblastoma. Plos One 2008; 3: 1-9.

21. Sałacińska-Łoś E, Kobos J, Taran K. Estimation of diagnostic value of chosen markers of neural differentiation in neuroblastoma group of tumors and pPNETs. Pol J Pathol 2008, 59: 195199.

22. Taran K, Kobos J, Sitkiewicz A, Sporny S. Estimation of prognostic value of CD44 expression in neuroblastic tumors in children. Folia Neuropathologica 2007; 45: 126-132.

\section{Address for correspondence}

Katarzyna Taran MD, PhD

Department of Pathology

Medical University of Lodz

Pomorska 251

92-213 Lodz, Poland

e-mail: dr.taran.patho@gmail.com 